\title{
Central Areolar Choroidal Dystrophy (CACD) and Age-Related Macular Degeneration (AMD): Differentiating Characteristics in Multimodal Imaging
}

\author{
Dženita Smailbodzic, ${ }^{1,2}$ Monika Fleckenstein, ${ }^{2,3}$ Thomas Theelen, ${ }^{1}$ Camiel J. F. Boon, ${ }^{1}$ \\ Ramon A. C. van Huet, ${ }^{1}$ Johannes P. H. van de Ven, ${ }^{1}$ Anneke I. Den Hollander, ${ }^{1,4}$ \\ Steffen Schmitz-Valckenberg, ${ }^{3}$ Carel B. Hoyng, ${ }^{1}$ Bernhard H. F. Weber, ${ }^{5}$ Frank G. Holz, ${ }^{3}$ \\ and B. Jeroen Klevering ${ }^{1}$
}

\begin{abstract}
PuRPose. Late-onset central areolar choroidal dystrophy (CACD) may easily be confused with geographic atrophy (GA) in AMD. To detect discerning features, the morphologic changes in CACD patients and in AMD patients were assessed with confocal scanning laser ophthalmoscopy (cSLO), fundus autofluorescence (FAF), and spectral-domain optical coherence tomography (SD-OCT).
\end{abstract}

Methods. A total of 30 CACD patients with identified PRPH2 gene mutations were analyzed and compared to 19 patients with early AMD and 13 patients with AMD-associated GA. The presence of drusen and pigment clumping was determined with color fundus photography. High-resolution in vivo imaging was performed with cSLO and SD-OCT. FAF images and SD-OCT volume scans were analyzed in each study eye.

Results. On FAF, a speckled FAF pattern occurred significantly more often in CACD (85\%) than in early AMD $(5.6 \% ; P<$ $0.0001)$. There was a significantly higher frequency of sub-RPE deposits in eyes with AMD than in eyes with CACD (36.8\% versus $2.1 \%$ of scans, $P=0.0019$ ). Reticular drusen could be visualized by SD-OCT and FAF imaging in $52.6 \%$ of the eyes

From the Departments of ${ }^{1}$ Ophthalmology and ${ }^{4} \mathrm{Human}$ Genetics, Radboud University Nijmegen Medical Centre, Nijmegen, The Netherlands; the ${ }^{3}$ Grade-Reading-Center, Department of Ophthalmology, University of Bonn, Bonn, Germany; and the ${ }^{5}$ Institute of Human Genetics, University of Regensburg, Regensburg, Germany.

${ }^{2}$ These authors contributed equally to the work presented here and should therefore be regarded as equivalent authors.

Supported by The Netherlands Organisation for Scientific Research Grant 016.096.309; the MD fonds; Oogfonds; Landelijke Stichting voor Blinden en Slechtzienden; Algemene Nederlandse Vereniging ter Voorkoming van Blindheid; Stichting Researchfonds Oogheelkunde; Stichting Nederlands Oogheelkundig Onderzoek; Stichting Blindenhulp; and the Gelderse Blindenstichting; and DFG (German Research Council) Grants Ho1926/3-1, WE 1259/19-2, and BONFOR 0-137-0012 (MF). The sponsor or funding organization had no role in the design or conduct of this research.

Submitted for publication May 23, 2011; revised September 13, 2011; accepted October 3, 2011.

Disclosure: D. Smailhodzic, None; M. Fleckenstein, Heidelberg Engineering (F, C); T. Theelen, None; C.J.F. Boon, None; R.A.C. van Huet, None; J.P.H. van de Ven, None; A.I. Den Hollander, None; $\mathbf{S}$. Schmitz-Valckenberg, Heidelberg Engineering (F, C), Optos (F, C), Topcon UK (F); C. B. Hoyng, None; B.H. F. Weber, None; F.G. Holz, Heidelberg Engineering (F, C), Optos (C), Carl Zeiss Meditec (C); B.J. Klevering, None

Corresponding author: Dženita Smailhodzic, Department of Ophthalmology, Radboud University Nijmegen Medical Centre, Philips van Leydenlaan 15, 6526 EX Nijmegen, The Netherlands;

d.smailhodzic@ohk.umcn.nl. with early AMD and in 100\% of the eyes with GA, whereas this drusen phenotype did not manifest in eyes with CACD.

Conclusions. Although outer retinal atrophy is the clinically common feature in advanced CACD as well as GA, there are microstructural alterations on high-resolution SD-OCT and FAF imaging that allow for the differentiation between CACD and AMD. The findings may help to identify patients in whom a diagnostic PRPH2 screening is warranted. (ClinicalTrials.gov number, NCT00393692.) (Invest Opbthalmol Vis Sci. 2011;52: 8908-8918) DOI:10.1167/iovs.11-7926

$\mathrm{C}$ entral areolar choroidal dystrophy (CACD) is a hereditary retinal disorder that affects the macula, resulting in progressive and usually profound visual loss. The hallmark feature of the disorder is a well-defined atrophy of the retinal pigment epithelium (RPE) and the choriocapillaris. ${ }^{1}$ Four clinical stages of the diseases have been described. ${ }^{2}$ In stage 1 CACD, subtle focal parafoveal pigmentary RPE changes can be observed on ophthalmoscopy. A typical stage 2 finding in the color image is an oval-to-round, mildly atrophic, hypopigmented area. This area, on a fundus autofluorescence (FAF) image, shows increased as well as decreased reflectivity resulting in a speckled FAF pattern. Stage 3 is characterized by one or more patches of well-demarcated RPE atrophy outside the fovea. In stage 4, the atrophic area involves the fovea, resulting in a markedly decreased visual acuity. ${ }^{2,3}$

Autosomal dominant CACD is most commonly caused by mutations in the peripherin-2 (PRPH2) gene (formerly known as peripherin/RDS). ${ }^{4,5}$ More than 90 different PRPH2 mutations associated with a wide spectrum of fundus alterations have been reported. To date, seven different mutations in the PRPH 2 gene have been identified to cause the CACD phenotype. $^{6-12}$ It may be challenging to diagnose CACD in the early stages of the disorder because of the relative nonspecific RPE abnormalities. Also, the late-onset variant may easily be confused with age-related macular degeneration (AMD) and thus be misdiagnosed.

AMD is a complex disease and as such is the result of multiple factors, some genetic and some exogenous. ${ }^{13}$ The early and intermediate stages of AMD are characterized by the presence of drusen and pigmentary changes. Choroidal neovascularization $(\mathrm{CNV})$ is present in the exudative form while the advanced nonexudative form (geographic atrophy, GA) is characterized by atrophy of the retinal pigment epithelium (RPE), the photoreceptors, and the choriocapillaris. Approximately $20 \%$ of AMD patients with severe visual impairment have lost central vision due to GA. ${ }^{14-18}$ Major genetic risk factors for AMD are certain common variants in the complement factor $\mathrm{H}(\mathrm{CFH})^{19-21}$ and the age-related maculopathy 
susceptibility 2 (ARMS2/LOC387715) genes. $^{22-24}$ Subsequently, other complement factor genes (i.e., $C 3, C F B / C 2$, and $C F I)$ were found to be associated with AMD. ${ }^{25-28}$ In contrast, a study involving 371 cases of AMD showed that the PRPH2 gene appears not to be involved in the pathogenesis of AMD. ${ }^{29}$ Despite these apparent differences in the underlying pathophysiological processes, CACD and AMD share many phenotypic characteristics. Up to a third of CACD patients develop visual loss at an older age, showing a considerable overlap with age of onset in AMD. ${ }^{3}$ Besides the circumscribed atrophic lesions that characterize the late stages in both disorders, drusenlike deposits have been described in a limited number of patients with CACD. ${ }^{30}$ In addition, the penetrance of CACD may be low (up to $21 \%$ nonpenetrance), which may mask the autosomal dominant mode of inheritance in some families and may further impede the correct diagnosis. ${ }^{3}$

In the present study, we analyzed the morphologic findings of 30 genetically confirmed CACD patients and a representative cohort of patients with early- and late-stage nonexudative AMD, to detect discerning features on ophthalmoscopy, FAF, and spectral-domain (SD) optical coherence tomography (OCT).

\section{Patients and Methods}

First, we evaluated 60 eyes of 30 patients with genetically proven CACD in various stages of the disease. All patients with CACD were randomly chosen from a large CACD cohort and examined at the Departments of Ophthalmology, Radboud University Nijmegen Medical Centre (Nijmegen, The Netherlands). Twenty-nine CACD patients carried the p.Arg142Trp PRPH2 mutation and one patient carried the p.Arg172Gln PRPH2 mutation. Both mutations were previously associated with CACD. ${ }^{6,31}$ Subsequently, to detect discerning features between the CACD phonotype and AMD we stratified CACD eyes into (1) early nonatrophic stages (CACD stages 1 and 2) and (2) late atrophic stages (CACD stages 3 and 4). Only one eye of each patient was included into the statistic analysis, resulting in 20 CACD eyes in group 1 and 18 eyes in group 2.

To compare the CACD phenotype with that of AMD, we evaluated 19 eyes (19 patients) with early AMD and 13 eyes (13 patients) with GA due to AMD. The diagnosis and grading of AMD was based on an international classification and grading system for age-related maculopathy and age-related macular degeneration, as described previously. ${ }^{32}$ AMD patients were examined at the Department of Ophthalmology, University of Bonn, Germany. All patients with AMD were participants of the multicenter, prospective FAM (Fundus Autofluorescence in Age-related Macular Degeneration) study. Eyes with hyperpigmentary changes and/or soft drusen without any sign of active or previous neovascular AMD or GA were included in the study. For comparison with late CACD stages, eyes with GA due to AMD were analyzed. These included eyes with FAF patterns similar to the speckled CACD FAF pattern in the perilesional zone of GA (i.e., the diffuse fine granular, the diffuse branching, and the diffuse trickling FAF phenotype; according to Holz et al. ${ }^{33}$ ) and without signs of active or previous neovascular AMD. Only one eye of each patient was included in the analysis, but in cases of bilateral early AMD with bilateral GA, only the right eye was included.

\section{Genetic Analysis}

The 32 AMD patients were analyzed for the presence of the PRPH2 p.Arg142Trp and the p.Arg172GIn mutations by direct Sanger sequencing. Genomic DNA was isolated from peripheral blood lymphocytes using standard extraction procedures. The c. $424 \mathrm{C}>\mathrm{T}$ (p.Arg142Trp) and c.515G $>$ A (p.Arg172Gln) positions in exon 1 of the PRPH 2 gene were inspected by using the oligonucleotide primers RDS.cEx01-F (5'-CTG CAC TTT TCC CAA GGC CCT AAG TC-3') and RDS.cEx01-R (5'-TGT CCC CAA TAT ATT CAT AGC TCT GAC CC-3') to PCR amplify the exonic fragment from the patient DNAs. Direct sequencing was then achieved with primers RDS.cEx01-R, RDS-ex1Fa (5'-AGC CAA GTA TGC CAG ATG GA-3'), or RDS-ex1Ra (5'-AGC AGA AAG CAG CAG AGA GC-3') by dye-termination chemistry (Big Dye Terminator Cycle Sequencing Kit, ver. 1.1; Applied Biosystems, Darmstadt, Germany). Reactions were analyzed with a sequencer (Prism Model 3130xl; Applied Biosystems). The study was performed in accordance with the tenets of the Declaration of Helsinki (1983 revision) and in accordance with the Medical Research Involving Human Subjects Act (WMO). The approval of the local ethics committee was obtained for both centers and a written informed consent to participate in this study was acquired from all subjects.

\section{Image Acquisition and Data Analysis}

Pupillary dilatation was achieved with topical $1.0 \%$ tropicamide and $2.5 \%$ phenylephrine before retinal imaging. Each participant underwent digital color fundus photography (Imagenet; Topcon Corporation, Tokyo, Japan [used in Nijmegen]; FF 450 Visupac ZK5; Carl Zeiss Meditec AG, Jena, Germany [used in Bonn]). High-resolution in vivo imaging was performed with a combined instrument (Spectralis HRA+ OCT, Heidelberg Engineering, Heidelberg, Germany) that allows for simultaneous recording of SD-OCT and cSLO (confocal scanning laser ophthalmoscope) as described previously. ${ }^{34}$ The cSLO images were obtained according to a standardized operation protocol as describe before. ${ }^{34,35}$ Nineteen horizontal SD-OCT scans through the macula area with simultaneous cSLO imaging were obtained for each study eye. Individual bands lying below the hyporeflective band of the outer nuclear layer $(\mathrm{ONL})$ were identified based on recent descriptions, as depictured in Figure $1 .^{36}$ Color fundus photography was used to identify the presence of drusen and pigmentary changes. FAF images were used to identify CACD stages as described previously. ${ }^{3}$ In AMD patients, FAF images were analyzed according to the classification of FAF patterns in early $\mathrm{AMD}^{37}$ and of abnormal FAF patterns in the junctional zone of GA, respectively. ${ }^{33}$

Microstructural changes were analyzed and categorized in consensus meetings of the authors. Subsequently, two graders (DS and MF) performed qualitative and quantitative analysis on fundus photography, FAF and SD-OCT images (19 single SD-OCT scans per study eye). Quantitative differences in the presence of retinal features were analyzed using Fisher's exact test and for the continuous variables, we used the Mann-Whitney $\mathrm{U}$ test. To adjust the $P$-value for multiple testing, we performed the Bonferroni correction. Reported $P$-values

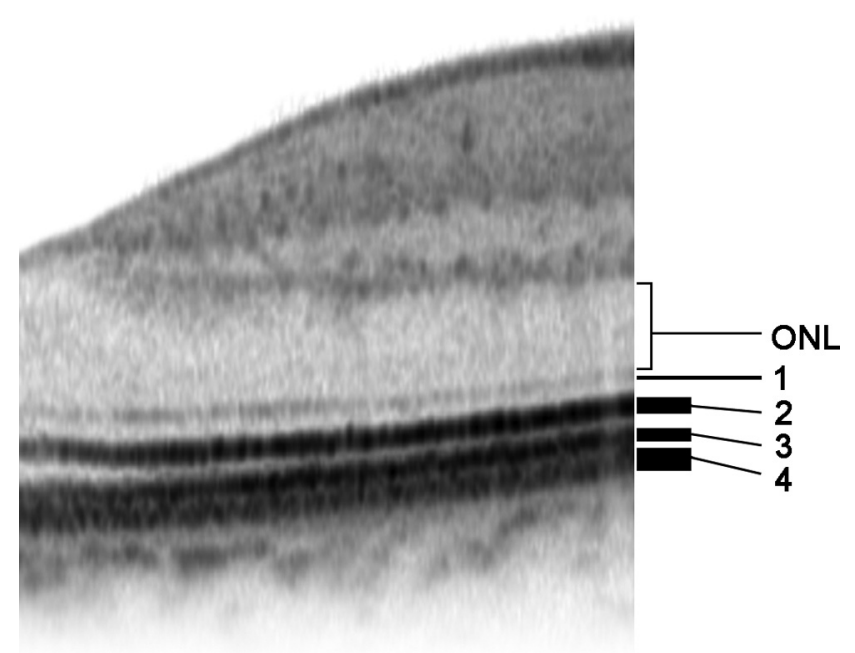

Figure 1. SD-OCT scan through the fovea of a normal retina of a 65-year-old male. Different hyperreflective bands can be defined that appear to correlate with the anatomic layers of the outer retina. ${ }^{36}$ Layers of the ONL: (1) ELM; (2) IPRL; (3) outer segment-RPE interdigitation; and (4) RPE-Bruch's membrane complex (RPE/BM). 
are two sided and considered statistically significant if lower than 0.002 (all statistical analyses: SPSS, ver. 17.0; IBM SPSS, Chicago, IL).

\section{Results}

\section{Description of CACD Stages}

Stage 1 CACD. Five eyes of three patients, who were members of two families, had stage 1 CACD. The presence of focal parafoveal RPE changes in a predominantly normal retina characterizes this early stage (Fig. 2). FAF imaging revealed lipofuscin accumulation indicated by an increased signal of these parafoveal lesions (Fig 2B). Corresponding SD-OCT scans showed focal photoreceptor/RPE abnormalities, ranging from subtle disruption and elongation of the line representing the interface of the inner and outer segments of the photoreceptor layer (IPRL) to frank loss of the normal outer retinal anatomy (Figs. 2C, 2D).

Stage 2 CACD. Stage 2 CACD was diagnosed in 27 eyes of 18 patients received. In this stage, a round and poorly demarcated area of hypopigmentation was observed on color fundus photography (Fig. 3A). The most striking finding was a speckled FAF that covered the macula (Fig. 3B). SD-OCT scans of this area demonstrated a loss of reflectivity and interruption of the IPRL and, to a lesser extent, of the external limiting membrane (ELM) (Fig. 3C). In addition, this area showed thinning and increased irregularity of the ONL. At the central macula, relative preservation of the reflectivity of the outer photoreceptor segments was observed (Figs. 3C, 3D). In 11 eyes (40.7\%) focal elongation of the photoreceptor outer segments was present on the SD-OCT (Fig. 3D). In three eyes (two patients), slight detachment of the neurosensory retina was noted (Fig. 3E). Compared to stage 1, a high number of SD-OCT hyperreflective clumps were noted, generally localized above the level of the RPE. Approximately $85 \%$ of the clumps correlated with markedly increased FAF signals (see Figs. 4D-4F).

Stage 3 CACD. Fourteen eyes of 10 patients demonstrated well-defined atrophy not involving the macula and were thus classified with stage 3 CACD (Fig. 4). A typical speckled FAF pattern always surrounds the atrophic areas (Fig. 4B). As in stage 2, SD-OCT scans within speckled FAF revealed a reflectivity loss and interruption of IPRL (Fig. 4C). The atrophic borders were characterized by a relative sharp disruption of the RPE layer with ELM covering the borders (Figs. 4C, 4F). In four eyes (29\%) rosettelike structures located at the photoreceptor layer were observed near the border of the atrophic area (see Fig. 5D). Hyporeflective spaces, located mainly in the outer plexiform layer, were occasionally present (39\% of stages 3 and 4$)$.

Stage 4 CACD. Fourteen eyes of nine patients demonstrated central atrophic lesions characteristic for CACD stage 4 (Fig. 5). In areas of atrophy, virtually all outer retinal layers were absent (Fig. 5C). Rosettelike structures originating from the photoreceptor layer were observed in 11 (79\%) eyes near the border of the atrophic area (Fig. 5D). Within the atrophic zones $21 \%$ of eyes had clusters of hyperreflective material correlating with increased FAF.

\section{CACD versus AMD}

Patients with CACD were significantly younger than those with AMD: 53.2 (SD 14.1) years versus 77.1 (SD 7.93) years $(P<$ $0.0001)$. A positive family history for visual loss was significantly more common in CACD than in AMD (80\% vs. $9.4 \% ; P<$ 0.0001). None of the AMD patients carried the PRPH2 p.Arg142Trp or the p.Arg172Gln gene mutation. Table 1 provides an overview of differences between CACD and AMD patients.

CACD Stages 1 and 2 versus Early AMD. On FAF, a speckled pattern of increased and decreased FAF signal as describe before ${ }^{3}$ occurred significantly more often in CACD than in AMD (85\% vs. $5.6 \% ; P<0.0001)$. However, the speckled FAF pattern in early AMD as described by Bindewald et al. ${ }^{37}$ (Fig. 6) was not as well demarcated and was more irregularly shaped than in CACD. In eyes with early AMD, the abnormal FAF pattern frequently extended beyond the macula and covered the entire posterior fundus. This effect contrasted with the sharply demarcated, regularly oval-shaped, speckled FAF pattern that was usually confined to the central macular in CACD (Fig. 3 versus Fig. 6). On SD-OCT imaging, in early AMD,
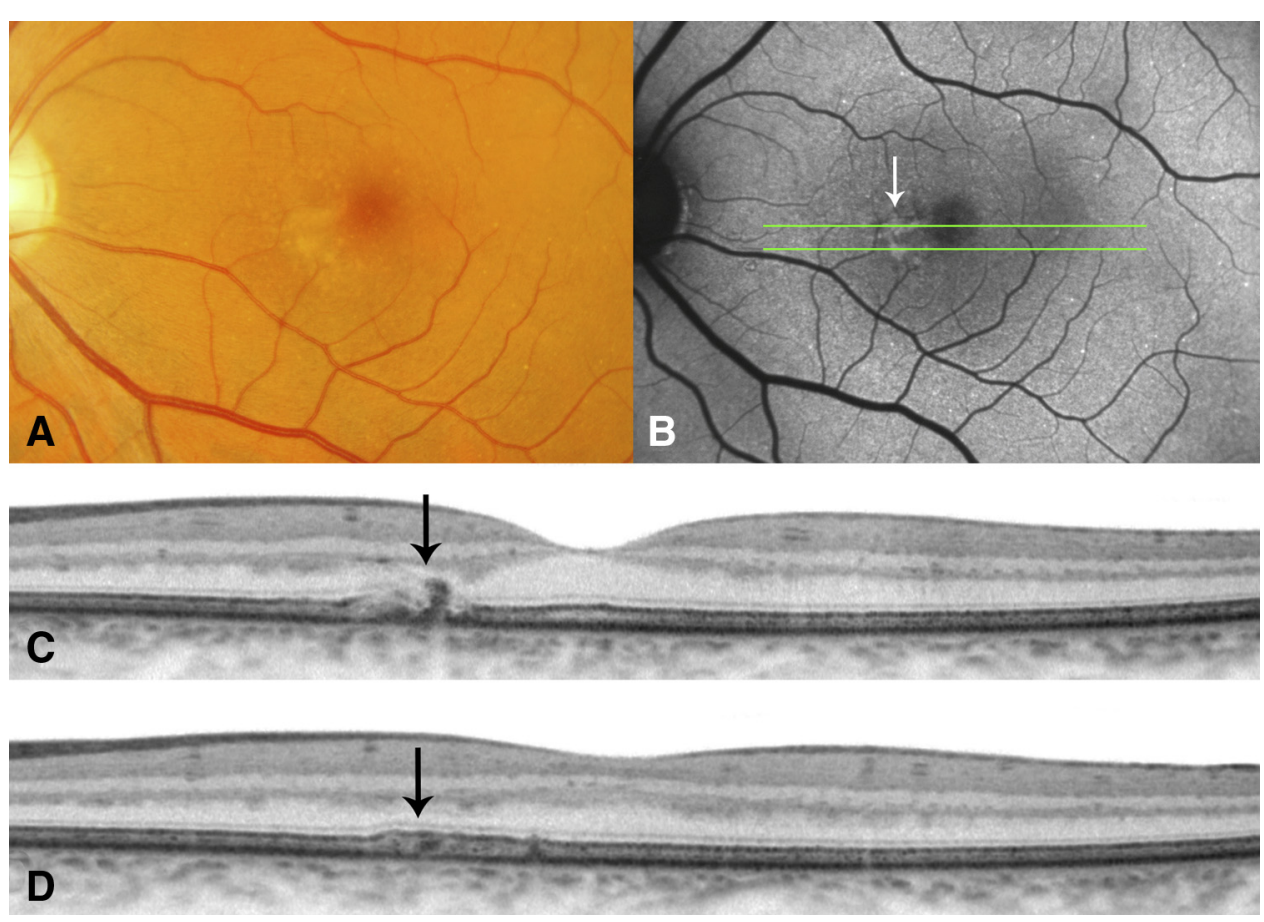

Figure 2. Stage 1 CACD. Green line: positions of the SD-OCT scans. Color fundus photography (A) showing a parafoveal increase in reflectivity, with some pigment clumping, co-located with a discrete increase of FAF (B). (C) Interruption of the normal anatomy of RPE and photoreceptor outer segments (arrow). (D) Subtle disruption, elongation, and reflectivity loss of IPRL and the RPEBruch's membrane complex on SD-OCT (arrow). 


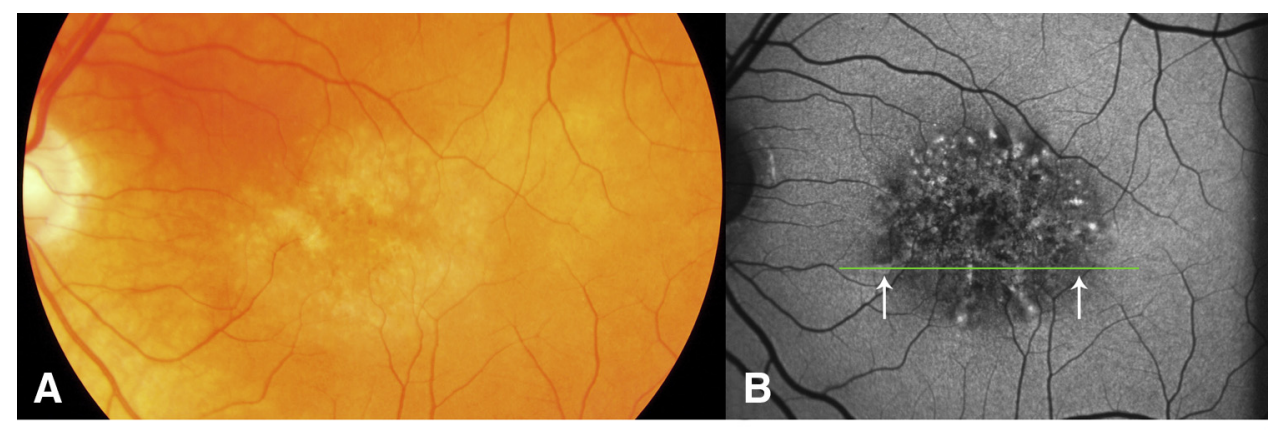

FIgURE 3. Stage 2 CACD. Green line: the position of the SD-OCT scans. Color fundus photography (A) shows a hypopigmented area, co-located with a speckled fundus autofluorescence appearance at the macula (B). SD-OCT shows reflectivity changes and interruptions of the IPRL and thinning of the ONL (C). White arrows $(\mathbf{B})$ indicate the area of changed SD-OCT between the black arrows in (C). In CACD stage 2 , elongation of the IPRL/RPE is visible in the transition zone (D, arrow). In late stage $2 \mathrm{CACD}$, interruption or even loss of the IPRL together with relatively preserved subfoveal reflectivity was noted, suggesting a partial detachment of the neurosensory retina $(\mathbf{E}$, arrow).

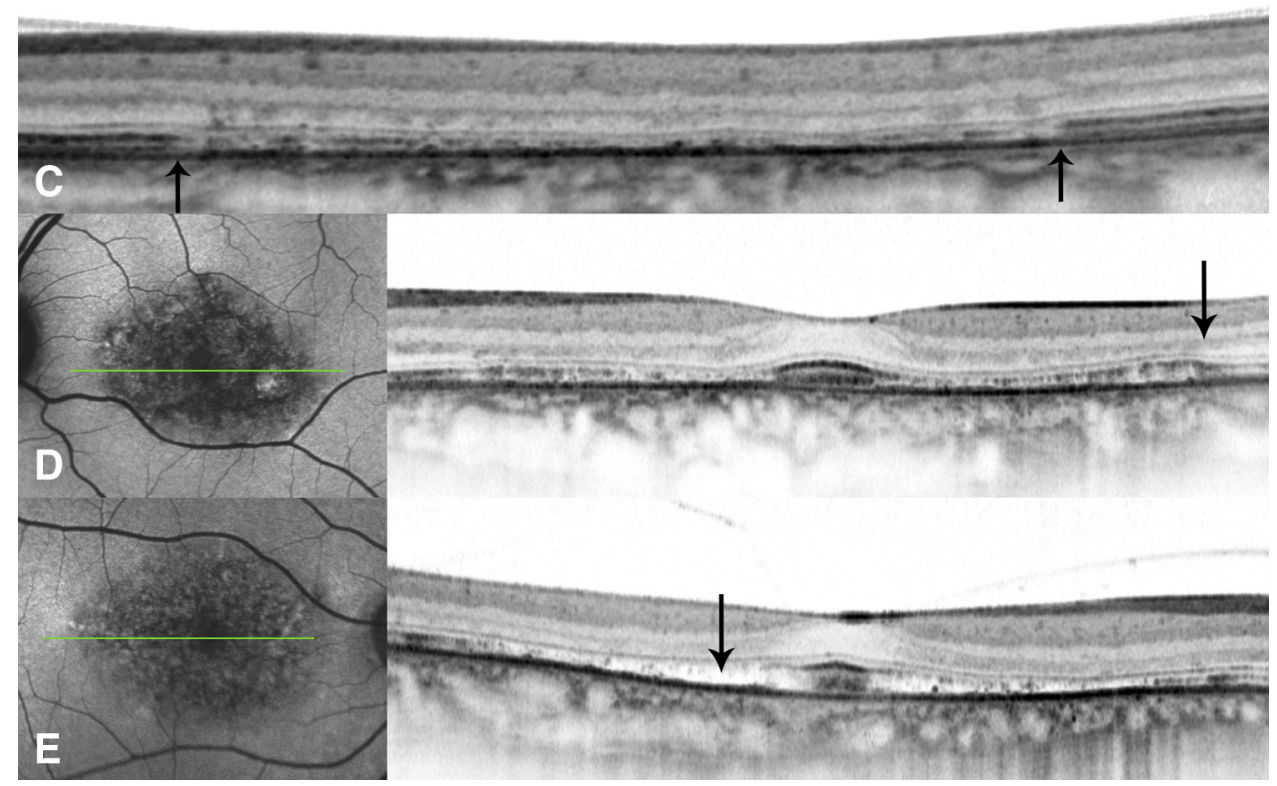

\section{Discussion}

This study was conducted to systematically analyze and compare the morphologic changes in the various stages of AMD and CACD. In accordance with the ophthalmoscopic similarities of these retinal disorders, SD-OCT and FAF findings also show that CACD shares many morphologic characteristics with atrophic AMD. These include hyperpigmentations, abnormal FAF and occurrence of atrophy of the outer retinal SD-OCT layers in the advanced stages. Nevertheless, there are morphologic differences that may help in the differentiation between CACD and AMD (Table 1).

In all but the initial stage of CACD, a speckled FAF pattern that was sharply demarcated from the surrounding retina, regularly oval shaped and confined to the central macular, was observed. In fact, this feature could be considered the hallmark of CACD stages 2, 3, and 4 and was the most prominent discriminating characteristic (Fig. 3). Although speckled FAF patterns previously have been described in AMD, ${ }^{33,37}$ the alterations were less regularly shaped, less well demarcated, and often extended beyond the macular area. In the speckled FAF area in CACD, SD-OCT revealed a corresponding disorganization of the photoreceptors and a loss of photoreceptor outer segments, illustrated by a disruption of the inner-outer photoreceptor junction accompanied by a thinning in the ONL. The predominant photoreceptor loss in CACD is a consequence of the underlying disease process. CACD is caused by a monogenetic defect in one of the two PRPH 2 genes and potential modifying environmental factors are thought to be of little relevance in this disorder. ${ }^{3}$ The Peripherin- 2 protein may function as an adhesion molecule involved in stabilization and 

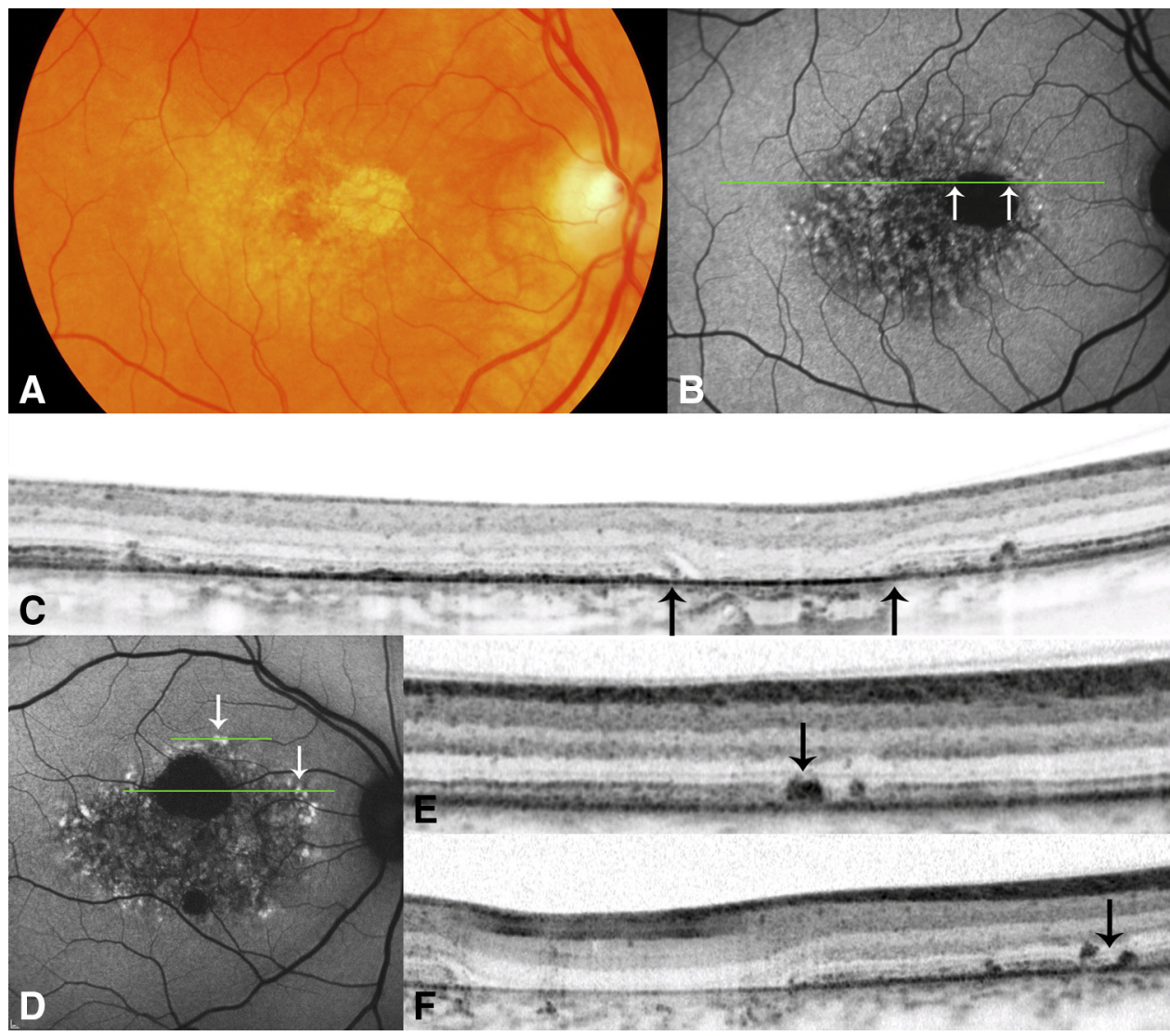

Figure 4. Stage 3 CACD. Green line: the position of the SD-OCT scans. Color fundus photography (A) shows an atrophy zone co-located with an absent FAF signal (B, arrows) embedded in a typical speckled FAF area. On SD-OCT (C), the atrophic area shows total absence of the outer retinal layers (within black arrows). The outer retinal changes within the zone of speckled FAF appears comparable with stage 2 CACD on SD-OCT. As in stage 2 CACD, hyperfluorescent spots on FAF (D, white arrows) co-localize with hyperreflective clumping on SD-OCT (E, F, black arrow). compaction of the outer segment discs and/or in the maintenance of the curvature of the rim of the discs. ${ }^{38-40}$ It has been demonstrated in a mouse model that one functional PRPH 2 allele is unable to support the normal outer segment morpho- genesis in cones and rods. ${ }^{41}$ Cones with one functional PRPH2 allele provide better sensitivity to light stimuli than those completely lacking PRPH2. These cones, however, are not as efficient in phototransduction as cones in wild-type mice. ${ }^{41}$ In
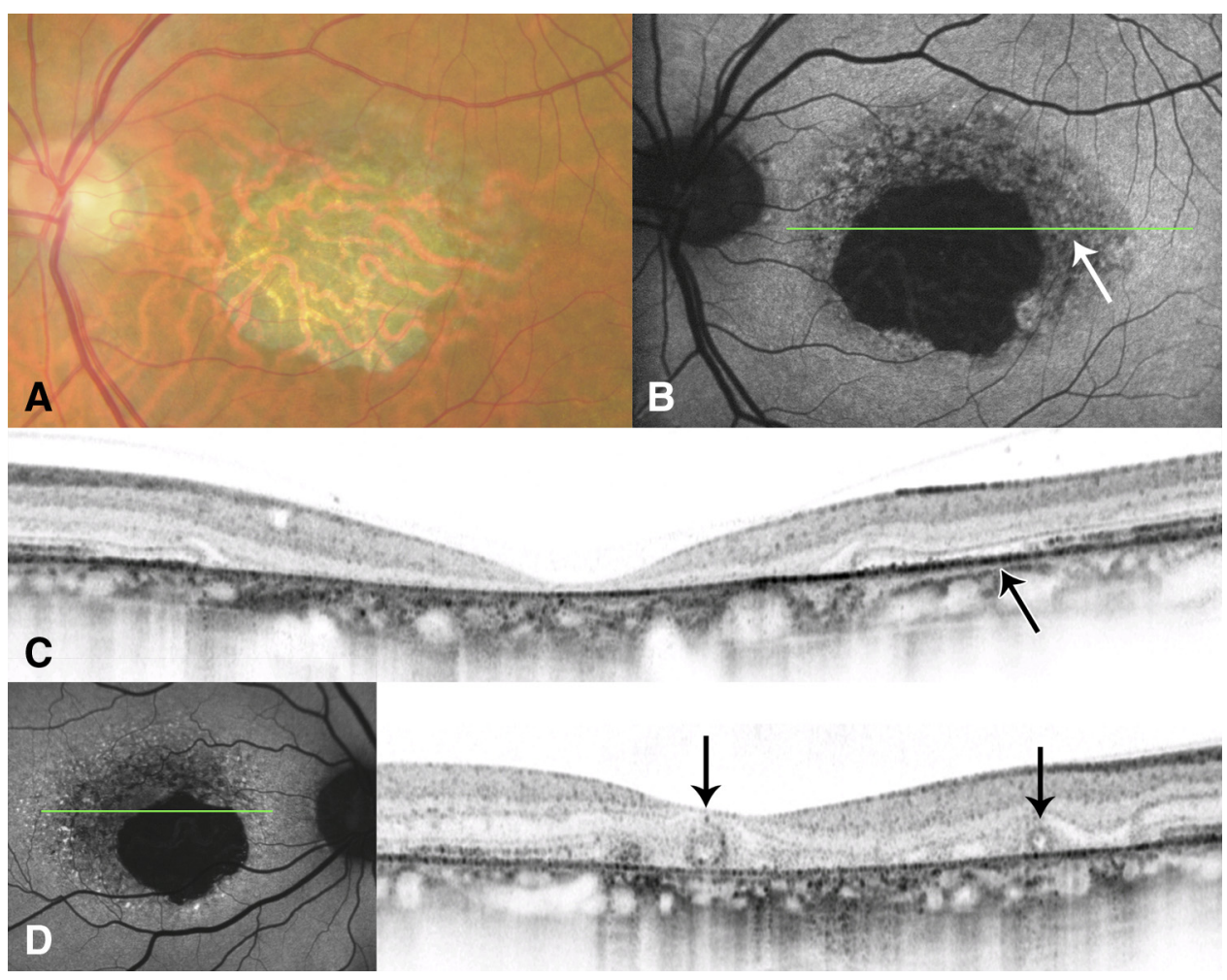

Figure 5. Stage 4 CACD. Green line: the position of the SD-OCT scans. Stage 4 CACD is characterized by atrophy of the fovea (A). As in early stages, a typically speckled autofluorescence with outer retinal changes on SD-OCT can be observed (B, C, arrows). In tangential SD-OCT scans through the margin of chorioretinal atrophy, rosettelike structures could be detected in many cases (D, arrows). 


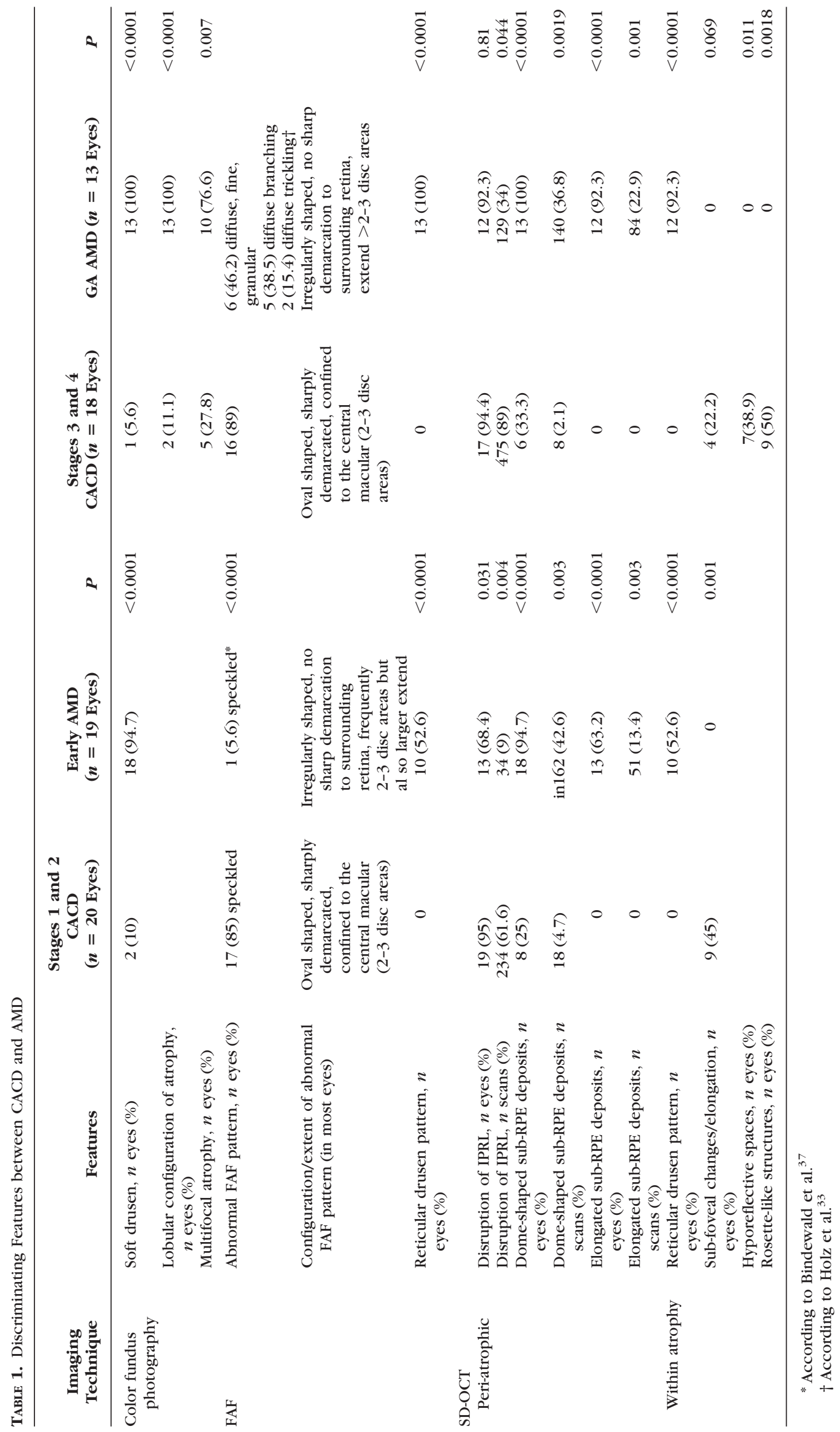




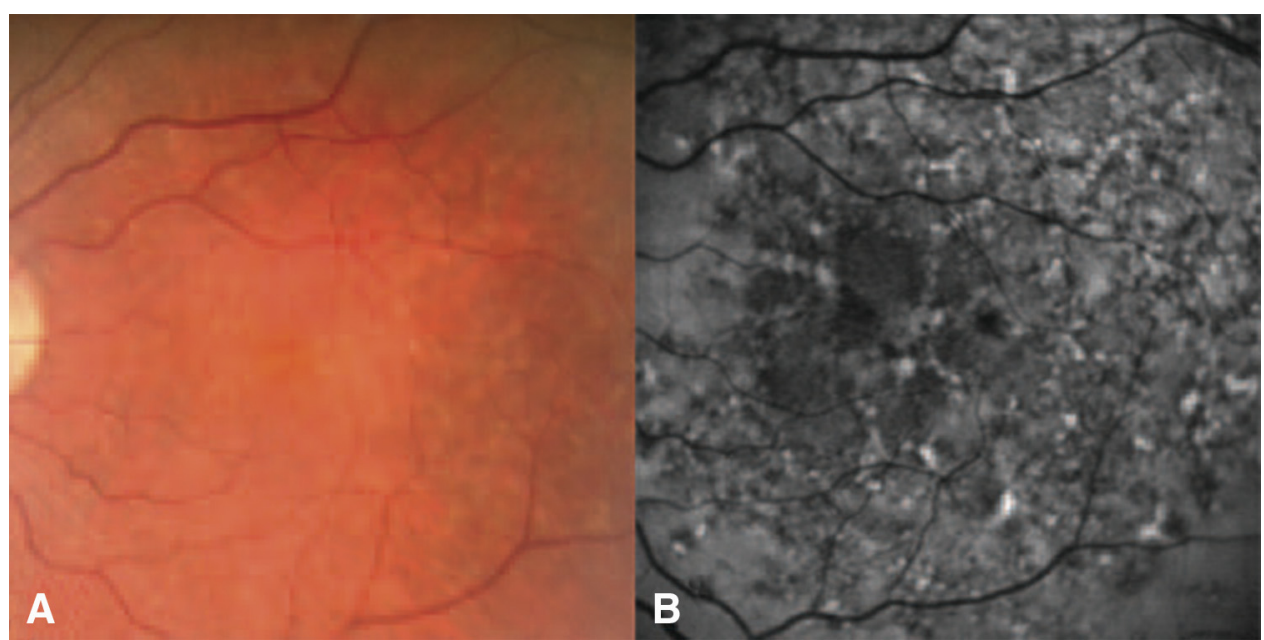

Figure 6. The speckled FAF pattern in early AMD. This FAF pattern (B) is characterized by the simultaneous presence of a variety of FAF abnormalities in a larger area of the FAF image. The changes may extend beyond the macular area and may cover the entire posterior fundus. Typically, these abnormalities include multiple small areas of irregularly increased and decreased FAF. The small areas of focal increased FAF may be punctuate or resemble linear structures. The corresponding abnormalities visible on color fundus photographs (A) include hyper- and hypopigmentation and multiple subconfluent and confluent drusen. Reprinted from Bindewald A, Bird AC, Dandekar SS, et al. Classification of fundus autofluorescence patterns in early age-related macular disease. Invest Ophthalmol Vis Sci. 2005;46(9):3309-3314. @ARVO.

human CACD patients, there appears to be no detectable early cone dysfunction, but, as in mice, the disturbance of outer segment morphogenesis may result in an increased phagocytosis. $^{42-44}$ The resulting accelerated lipofuscin accumulation in the RPE may cause progressive impartment of lysosomal functions and, ${ }^{42,45}$ over time, may contribute to RPE cell death. ${ }^{46}$ Increased levels of RPE lipofuscin have been measured in a CACD patient carrying the p.Arg172Trp point mutation. ${ }^{47,48}$ It is plausible that the typical speckled FAF in the posterior pole of CACD stages 2 to 4 represent simultaneous occurrence of lipofuscin accumulation and focal atrophy of the photoreceptor-RPE functional unit. A reduction in cone density within areas of speckled FAF has also been described in four patients with macular dystrophies caused by the different PRPH2 mutations. ${ }^{49}$ Interestingly, in CACD stages 2 and 3, a subfoveal reflectivity was often preserved, indicating that foveal cones may be less vulnerable to the effects of a PRPH2 mutation. Highly heterogeneous FAF patterns in patients with GA in AMD have been reported previously. ${ }^{49}$ In this study, we selected and studied those FAF patterns of AMD-associated GA that could be confused with speckled FAF pattern as observed in CACD.

Another observation in the CACD group constituted a rosettelike structure, mainly located at the border of the atrophic zone. These were not observed in the AMD group and appear to represent a morphologic feature associated with CACD. Rosettelike structures originating from photoreceptors have been observed in retinas of mice with one functional PRPH 2 allele. ${ }^{41}$ This histopathologic study indicated that the rosettes develop as a consequence of tensional forces exerted on cells by the ELM to overcome the excessive space and keep the photoreceptors close to each other. We hypothesize that our CACD population resembles this mouse model. However, this phenomenon is only observed in advanced CACD stages when the outer segments may lose their normal morphology and RPE apposition. Zweifel et al. ${ }^{50}$ described similar structural changes in the outer retinal layer, also in GA patients, and coined the term outer retinal tabulations. When we take their study into consideration, we must conclude that rosettelike structures are significantly more often present in CACD than in GA.

SD-OCT revealed sub-RPE deposits in eyes with AMD (Figs. $7,8)$, whereas these were extremely rare in CACD. We distin- guished two types of sub-RPE deposits: dome-shaped and elongated sub-RPE deposits. The dome-shaped deposits on SD-OCT correlated with funduscopically typical soft drusen while the elongated deposits had no clear funduscopic correlation, but were frequently associated with hyperpigmentary changes and a mildly increased FAF signal (Fig. 8). Within the elongated deposits, in most eyes a characteristic SD-OCT alteration was subtle splitting of the IPRL, but in eyes of the diffuse-trickling phenotype, it may be markedly pronounced (Fig. 8). ${ }^{51}$ The elongated deposits most likely correlate with basal laminar deposits (BLamD) histopathologically, which occur between the RPE basement membrane and the RPE plasma membrane. ${ }^{52-54}$ BLamD have been identified in human donor eyes as hallmarks of AMD, particularly in late-stage AMD. ${ }^{54-58}$ The higher prevalence of sub-RPE deposits in AMD patients was also associated with the late atrophic stage. The atrophic zones in GA were highly irregular and showed residual material on Bruch's membrane. In CACD, by contrast, the atrophic areas were rather smooth and homogeneous. Furthermore, reticular drusen frequently observed in AMD were never noted adjacent to or within the CACD lesions. The much higher occurrence of sub-RPE deposits, the reticular drusen, and the irregular structure of retinal layers within the atrophic lesion in AMD patients all indicate different pathogenetic mechanisms for CACD versus atrophic AMD although, the outer atrophy finally represents a common downstream pathogenetic pathway. CACD is a monogenetic disorder that appears to cause direct photoreceptor damage with subsequent RPE cell loss. AMD, on the other hand, is currently thought of as representing a complex, multifactorial disease characterized by initial (sub)-RPE alterations followed by photoreceptor and RPE cell loss and/or secondary angiogenic processes.

The prevalence of end-stage AMD has been estimated at $3 \%$ in people aged over 65 years, rising to $11 \%$ in those over 85 years. ${ }^{59}$ Therefore, AMD-mimicking diseases should be suspected in relatively young patients with atrophic lesions. It seems reasonable to assume that late-onset macular dystrophies such as CACD are underdiagnosed and confused with AMD on a regular basis-the main reasons being the low prevalence of macular dystrophies and their phenotypic similarities with AMD, especially in the absence of a positive 
FIGURE 7. Subretinal pigment epithelium deposits in early AMD. The dome-shaped elevations of the outer retinal layers in the SD-OCT scans (C-E) correspond to soft drusen on color fundus photography (A). Hyperpigmentations in the fundus image correspond to an increased FAF signal (B) and hyperreflective structures overlaying the drusen in the SD-OCT scan (D). The green line indicates the position of the SD-OCT scans.


Figure 8. Subretinal pigment epithelium deposits in early AMD. Elongated elevations of the outer retinal layers in the SD-OCT scan (C) may appear in areas with hyperpigmentation (A) and mildly increased FAF (B). Repeated fluorescein angiography showed no signs of CNV or scaring (data not shown). Green line: the position of the SD-OCT scans. In this eye, there is an extreme amount of deposit beneath the RPE. In most AMD eyes, this SD-OCT finding is more subtle.

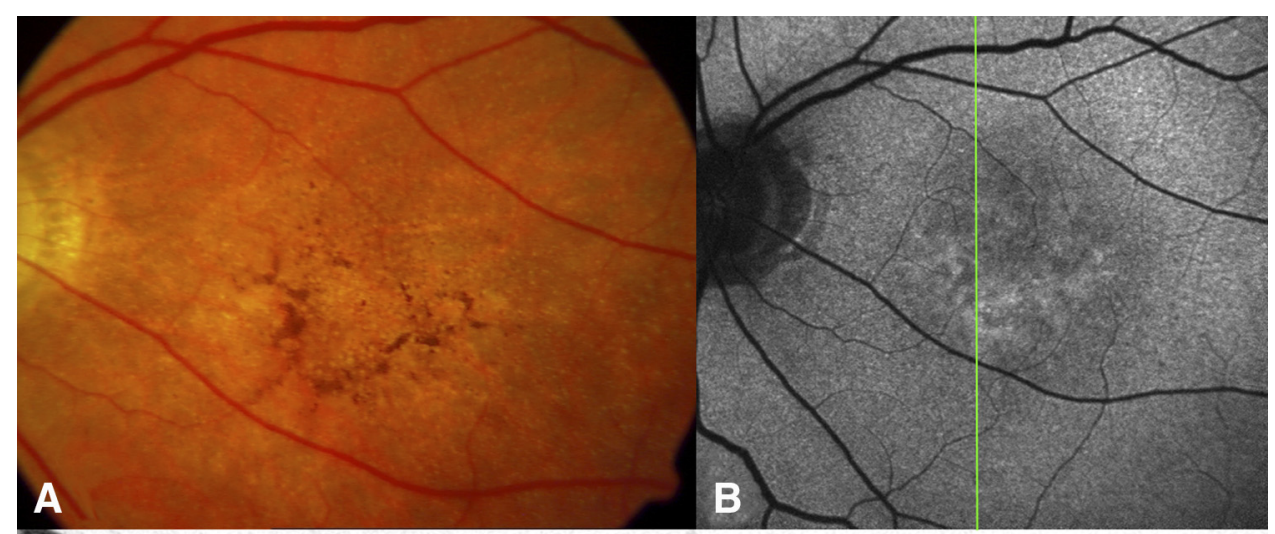




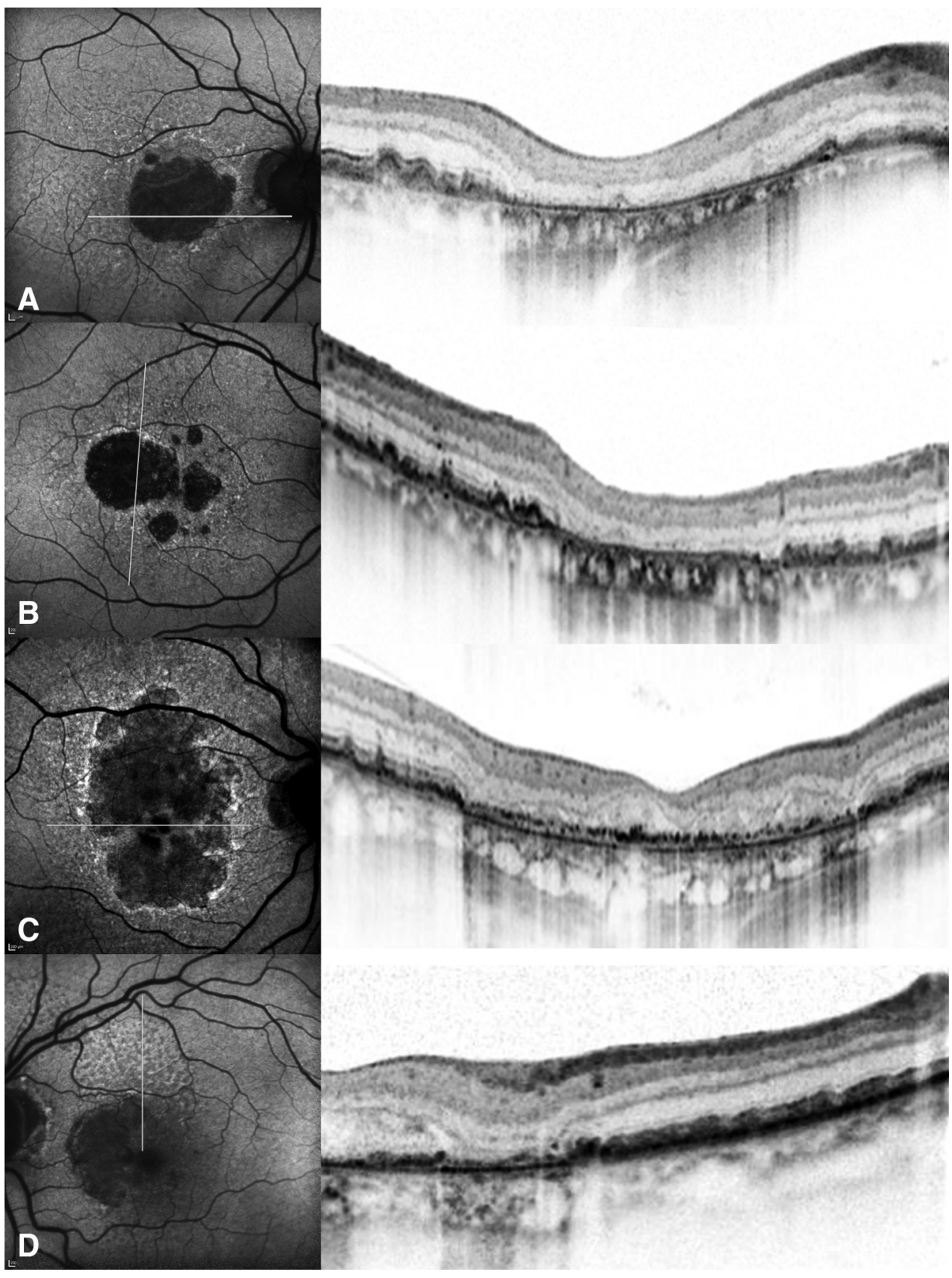

Figure 9. Simultaneous FAF and SD-OCT imaging in GA due to AMD. White line: the position of the SDOCT scans. (A) Diffuse branching, (B) diffuse fine granular, $(\mathbf{C}, \mathbf{D})$ and diffuse trickling FAF patterns in the peri-atrophic zone (according to Holz et al. ${ }^{33}$ ). SD-OCT imaging shows dome-shaped elevations (A, B) and elongated sub-RPE deposits (C) in the peri-atrophic area. (D) Reticular drusen pattern in FAF imaging with corresponding characteristic SD-OCT finding. Note the irregular structure of the remaining retinal layers within the atrophic lesion $(\mathbf{C}, \mathbf{D})$. family history. Therapeutic options for both disorders are currently limited; however, this could change in the near future. Oxidative stress is involved in AMD pathogenesis, and antioxidants, such as zinc and certain vitamins, may slow the progression of the disease. ${ }^{60}$ In the future, targeted manipulation of the alternative complement pathway, may provide a more powerful weapon in the battle against AMDrelated vision loss. ${ }^{61,62}$ In $\mathrm{CACD}$, the development of gene therapy, appears currently the most promising approach. The proper differentiation between AMD and CACD becomes of further consequence when the autosomal dominant inheritance of the latter is taken into consideration, as well as the differences in visual prognosis.

This study identified several morphologic distinguishing features between CACD and AMD based on noninvasive novel imaging tools (i.e., SD-OCT and FAF). These findings may aid the clinician in discerning these entities and may help in the identification of patients where genetic analysis for verification of a PRPH2 mutation is warranted.

\section{Acknowledgments}

The authors thank Kerstin Meier for help with the molecular analysis.

\section{References}

1. Ashton N. Central areolar choroidal sclerosis; a histo-pathological study. Br J Ophthalmol. 1953;37:140-147.

2. Hoyng CB, Deutman AF. The development of central areolar choroidal dystrophy. Graefes Arch Clin Exp Ophthalmol. 1996;234: 87-93.

3. Boon CJ, Klevering BJ, Cremers FP, et al. Central areolar choroidal dystrophy. Ophthalmology. 2009;116:771-782.

4. Gamundi MJ, Hernan I, Muntanyola M, et al. High prevalence of mutations in peripherin/RDS in autosomal dominant macular dystrophies in a Spanish population. Mol Vis. 2007;13:1031-1037. 
5. Hughes AE, Lotery AJ, Silvestri G. Fine localisation of the gene for central areolar choroidal dystrophy on chromosome $17 \mathrm{p} . \mathrm{J}$ Med Genet. 1998;35:770-772.

6. Hoyng CB, Heutink P, Testers L, Pinckers A, Deutman AF, Oostra BA. Autosomal dominant central areolar choroidal dystrophy caused by a mutation in codon 142 in the peripherin/RDS gene. Am J Ophthalmol. 1996;121:623-629.

7. Kohl S, Christ-Adler M, Apfelstedt-Sylla E, et al. RDS/peripherin gene mutations are frequent causes of central retinal dystrophies. J Med Genet. 1997;34:620-626.

8. Nichols BE, Sheffield VC, Vandenburgh K, Drack AV, Kimura AE, Stone EM. Butterfly-shaped pigment dystrophy of the fovea caused by a point mutation in codon 167 of the RDS gene. Nat Genet. 1993;3:202-207.

9. Reig C, Serra A, Gean E, et al. A point mutation in the RDSperipherin gene in a Spanish family with central areolar choroidal dystrophy. Ophthalmic Genet. 1995;16:39-44.

10. Schatz H. Diagnostic and therapeutic challenges. Retina. 2003;23: $530-535$.

11. Trujillo MJ, Bueno J, Osorio A, et al. Three novel RDS-peripherin mutations (689delT, 857del17, G208D) in Spanish families affected with autosomal dominant retinal degenerations: mutations in brief no 147 online. Hum Mutat. 1998;12:70.

12. Yanagihashi S, Nakazawa M, Kurotaki J, Sato M, Miyagawa Y, Ohguro H. Autosomal dominant central areolar choroidal dystrophy and a novel Arg195Leu mutation in the peripherin/RDS gene. Arch Opbthalmol. 2003;121:1458-1461.

13. de Jong PT. Age-related macular degeneration. $N$ Engl J Med 2006;355:1474-1485.

14. Friedman DS, O'Colmain BJ, Munoz B, et al. Prevalence of agerelated macular degeneration in the United States. Arch Opbthalmol. 2004;122:564-572.

15. Klaver CC, Wolfs RC, Vingerling JR, Hofman A, de Jong PT. Agespecific prevalence and causes of blindness and visual impairment in an older population: the Rotterdam Study. Arch Opbthalmol. 1998; 116:653-658.

16. Klein R, Klein BE, Tomany SC, Meuer SM, Huang GH. Ten-year incidence and progression of age-related maculopathy: The Beaver Dam eye study. Ophthalmology. 2002;109:1767-1779.

17. Mitchell P, Smith W, Attebo K, Wang JJ. Prevalence of age-related maculopathy in Australia. The Blue Mountains Eye Study. Opbthalmology. 1995;102:1450-1460.

18. Sunness JS, Gonzalez-Baron J, Applegate CA, et al. Enlargement of atrophy and visual acuity loss in the geographic atrophy form of age-related macular degeneration. Ophthalmology. 1999;106: $1768-1779$

19. Edwards AO, Ritter R III, Abel KJ, Manning A, Panhuysen C, Farrer LA. Complement factor $\mathrm{H}$ polymorphism and age-related macular degeneration. Science. 2005;308:421-424.

20. Hageman GS, Anderson DH, Johnson LV, et al. A common haplotype in the complement regulatory gene factor $\mathrm{H}(\mathrm{HF} 1 / \mathrm{CFH})$ predisposes individuals to age-related macular degeneration. Proc Natl Acad Sci U S A. 2005;102:7227-7232.

21. Haines JL, Hauser MA, Schmidt S, et al. Complement factor $\mathrm{H}$ variant increases the risk of age-related macular degeneration. Science. 2005;308:419-421.

22. Fisher SA, Abecasis GR, Yashar BM, et al. Meta-analysis of genome scans of age-related macular degeneration. Hum Mol Genet. 2005; 14:2257-2264.

23. Seddon JM, Santangelo SL, Book K, Chong S, Cote J. A genomewide scan for age-related macular degeneration provides evidence for linkage to several chromosomal regions. Am J Hum Genet. 2003;73:780-790.

24. Weeks DE, Conley YP, Tsai HJ, et al. Age-related maculopathy: an expanded genome-wide scan with evidence of susceptibility loci within the 1q31 and 17q25 regions. Am J Opbthalmol. 2001;132 682-692.

25. Fagerness JA, Maller JB, Neale BM, Reynolds RC, Daly MJ, Seddon JM. Variation near complement factor $\mathrm{I}$ is associated with risk of advanced AMD. Eur J Hum Genet. 2009;17:100-104.

26. Gold B, Merriam JE, Zernant J, et al. Variation in factor B (BF) and complement component 2 (C2) genes is associated with agerelated macular degeneration. Nat Genet. 2006;38:458-462.
27. Maller JB, Fagerness JA, Reynolds RC, Neale BM, Daly MJ, Seddon JM. Variation in complement factor 3 is associated with risk of age-related macular degeneration. Nat Genet. 2007;39:1200-1201.

28. Yates JR, Sepp T, Matharu BK, et al. Complement C3 variant and the risk of age-related macular degeneration. $N$ Engl J Med. 2007; 357:553-561.

29. Baird PN, Richardson A, Islam A, Lim L, Guymer R. Analysis of the RDS/peripherin gene in age-related macular degeneration. Clin Exp Opbthalmol. 2007;35:194-195.

30. Klevering BJ, van DM, van Hogerwou AJ, et al. Central areolar choroidal dystrophy associated with dominantly inherited drusen. Br J Opbthalmol. 2002;86:91-96.

31. Wroblewski JJ, Wells JA III, Eckstein A, et al. Macular dystrophy associated with mutations at codon 172 in the human retinal degeneration slow gene. Opbthalmology. 1994;101:12-22.

32. Bird AC, Bressler NM, Bressler SB, et al. An international classification and grading system for age-related maculopathy and agerelated macular degeneration. The International ARM Epidemiological Study Group. Surv Ophthalmol. 1995;39:367-374.

33. Holz FG, Bindewald-Wittich A, Fleckenstein M, Dreyhaupt J, Scholl HP, Schmitz-Valckenberg S. Progression of geographic atrophy and impact of fundus autofluorescence patterns in age-related macular degeneration. Am J Opbthalmol. 2007;143:463-472.

34. Helb HM, Charbel Issa P, Fleckenstein M, et al. Clinical evaluation of simultaneous confocal scanning laser ophthalmoscopy imaging combined with high-resolution, spectral-domain optical coherence tomography. Acta Opbthalmol. 2010;88:842-849.

35. Fleckenstein M, Charbel IP, Helb HM, et al. High-resolution spectral domain-OCT imaging in geographic atrophy associated with age-related macular degeneration. Invest Ophthalmol Vis Sci. 2008;49:4137-4144.

36. Pircher M, Gotzinger E, Findl O, et al. Human macula investigated in vivo with polarization-sensitive optical coherence tomography. Invest Ophthalmol Vis Sci. 2006;47:5487-5494.

37. Bindewald A, Bird AC, Dandekar SS, et al. Classification of fundus autofluorescence patterns in early age-related macular disease. Invest Ophthalmol Vis Sci. 2005;46:3309-3314.

38. Arikawa K, Molday LL, Molday RS, Williams DS. Localization of peripherin/rds in the disk membranes of cone and rod photoreceptors: relationship to disk membrane morphogenesis and retinal degeneration. J Cell Biol. 1992;116:659-667.

39. Boon CJ, den Hollander AI, Hoyng CB, Cremers FP, Klevering BJ, Keunen JE. The spectrum of retinal dystrophies caused by mutations in the peripherin/RDS gene. Prog Retin Eye Res. 2008;27: 213-235.

40. Molday RS, Hicks D, Molday L. Peripherin. A rim-specific membrane protein of rod outer segment discs. Invest Ophthalmol Vis Sci. $1987 ; 28: 50-61$.

41. Farjo R, Fliesler SJ, Naash MI. Effect of Rds abundance on cone outer segment morphogenesis, photoreceptor gene expression, and outer limiting membrane integrity. J Comp Neurol. 2007;504: $619-630$

42. Davies S, Elliott MH, Floor E, et al. Photocytotoxicity of lipofuscin in human retinal pigment epithelial cells. Free Radic Biol Med. 2001;31:256-265.

43. Papermaster DS. The birth and death of photoreceptors: The Friedenwald Lecture. Invest Ophthalmol Vis Sci. 2002;43:13001309.

44. Usukura J, Bok D. Changes in the localization and content of opsin during retinal development in the rds mutant mouse: immunocytochemistry and immunoassay. Exp Eye Res. 1987;45:501-515.

45. Shamsi FA, Boulton M. Inhibition of RPE lysosomal and antioxidant activity by the age pigment lipofuscin. Invest Opbthalmol Vis Sci. 2001;42:3041-3046.

46. Sparrow JR, Boulton M. RPE lipofuscin and its role in retinal pathobiology. Exp Eye Res. 2005;80:595-606.

47. Downes SM, Fitzke FW, Holder GE, et al. Clinical features of codon 172 RDS macular dystrophy: similar phenotype in 12 families. Arch Opbthalmol. 1999;117(10):1373-1383.

48. von Rückmann RA, Fitzke FW, Bird AC. Distribution of pigment epithelium autofluorescence in retinal disease state recorded in vivo and its change over time. Graefes Arch Clin Exp Ophthalmol. 1999;237:1-9. 
49. Duncan JL, Talcott KE, Ratnam K, et al. Cone structure in retina degeneration associated with mutations in the peripherin/RDS gene. Invest Ophthalmol Vis Sci. 2011;52:1557-1566.

50. Zweifel SA, Engelbert M, Laud K, Margolis R, Spaide RF, Freund KB. Outer retinal tubulation: a novel optical coherence tomography finding. Arch Opbthalmol. 2009;127:1596-1602.

51. Fleckenstein M, Schmitz-Valckenberg S, Martens C, et al. Fundus autofluorescence and spectral-domain optical coherence tomography characteristics in a rapidly progressing form of geographic atrophy. Invest Ophthalmol Vis Sci. 2011;52:3761-3766.

52. Loffler KU, Lee WR. Basal linear deposit in the human macula Graefes Arch Clin Exp Opbthalmol. 1986;224:493-501.

53. Marshall GE, Konstas AG, Reid GG, Edwards JG, Lee WR. Type IV collagen and laminin in Bruch's membrane and basal linear deposit in the human macula. Br J Opbthalmol. 1992;76:607-614.

54. van der Schaft TL, Mooy CM, de Bruijn WC, de Jong PT. Early stages of age-related macular degeneration: an immunofluorescence and electron microscopy study. Br J Ophthalmol. 1993;77:657-661.

55. Curcio CA, Millican CL. Basal linear deposit and large drusen are specific for early age-related maculopathy. Arch Ophthalmol. 1999; 117:329-339.

56. Green WR, Enger C. Age-related macular degeneration histopathologic studies: the 1992 Lorenz E. Zimmerman Lecture. 1992. Ret ina. 2005;25(5 suppl):1519-1535.
57. Sarks SH. Ageing and degeneration in the macular region: a clinicopathological study. Br J Ophthalmol. 1976;60:324-341.

58. Spraul CW, Lang GE, Grossniklaus HE, Lang GK. Histologic and morphometric analysis of the choroid, Bruch's membrane, and retinal pigment epithelium in postmortem eyes with age-related macular degeneration and histologic examination of surgically excised choroidal neovascular membranes. Surv Opbthalmol. 1999;44(suppl 1):S10-S32.

59. Vingerling JR, Klaver CC, Hofman A, De Jong PT. Epidemiology of age-related maculopathy. Epidemiol Rev. 1995;17:347-360.

60. A randomized, placebo-controlled, clinical trial of high-dose supplementation with vitamins $\mathrm{C}$ and $\mathrm{E}$, beta carotene, and zinc for age-related macular degeneration and vision loss: AREDS report no. 8. Arch Opbthalmol. 2001;119:1417-1436.

61. Charbel IP, Chong NV, Scholl HP. The significance of the complement system for the pathogenesis of age-related macular degeneration: current evidence and translation into clinical application. Graefes Arch Clin Exp Opbthalmol. 2011;249:163174.

62. Rohrer B, Long Q, Coughlin B, et al. A targeted inhibitor of the alternative complement pathway reduces angiogenesis in a mouse model of age-related macular degeneration 19. Invest Ophthalmol Vis Sci. 2009;50:3056-3064. 